\title{
Responsiveness of PROMIS and Patient Health Questionnaire (PHQ) Depression Scales in three clinical trials
}

\author{
Kurt Kroenke ${ }^{1,2^{*}}$ (D), Timothy E. Stump ${ }^{3}$, Chen X. Chen ${ }^{4}$, Jacob Kean ${ }^{5}$, Teresa M. Damush ${ }^{1,2,6}$, Matthew J. Bair ${ }^{1,2,6}$, \\ Erin E. Krebs ${ }^{7,8}$ and Patrick O. Monahan ${ }^{3}$
}

\begin{abstract}
Background: The PROMIS depression scales are reliable and valid measures that have extensive normative data in general population samples. However, less is known about how responsive they are to detect change in clinical settings and how their responsiveness compares to legacy measures. The purpose of this study was to assess and compare the responsiveness of the PROMIS and Patient Health Questionnaire (PHQ) depression scales in three separate samples.
\end{abstract}

Methods: We used data from three clinical trials (two in patients with chronic pain and one in stroke survivors) totaling 651 participants. At both baseline and follow-up, participants completed four PROMIS depression fixed-length scales as well as legacy measures: Patient Health Questionnaire 9-item and 2-item scales (PHQ-9 and PHQ-2) and the SF-36 Mental Health scale. We measured global ratings of depression change, both prospectively and retrospectively, as anchors to classify patients as improved, unchanged, or worsened. Responsiveness was assessed with standardized response means, statistical tests comparing change groups, and area-under-curve analysis.

Results: The PROMIS depression and legacy scales had generally comparable responsiveness. Moreover, the four PROMIS depression scales of varying lengths were similarly responsive. In general, measures performed better in detecting depression improvement than depression worsening. For all measures, responsiveness varied based on the study sample and on whether depression improved or worsened.

Conclusions: Both PROMIS and PHQ depression scales are brief public domain measures that are responsive (i.e., sensitive to change) and thus appropriate as outcome measures in research as well as for monitoring treatment in clinical practice.

Trial registration ClinicalTrials.gov ID: NCT01236521, NCT01583985, NCT01507688

Keywords: PROMIS, PHQ-9, Depression, Responsiveness, Sensitivity to change, Psychometrics

\section{Background}

Depression is the most common mental health disorder in both clinical practice and the general population, a major contributor to disability and health care costs, and

*Correspondence: kkroenke@regenstrief.org

1 Department of Medicine, Indiana University School of Medicine, Indianapolis, IN, USA

Full list of author information is available at the end of the article an important cause of morbidity as well as early mortality [1]. Because the assessment and monitoring of depression relies principally on patient-reported symptoms, reliable and valid scales are essential for both research and clinical practice. The National Institutes of Health has made substantial investments in developing and testing the Patient-Reported Outcomes Measurement Information System (PROMIS) measures to assess symptoms original author(s) and the source, provide a link to the Creative Commons licence, and indicate if changes were made. The images or other third party material in this article are included in the article's Creative Commons licence, unless indicated otherwise in a credit line to the material. If material is not included in the article's Creative Commons licence and your intended use is not permitted by statutory regulation or exceeds the permitted use, you will need to obtain permission directly from the copyright holder. To view a copy of this licence, visit http://creativecommons.org/licenses/by/4.0/. The Creative Commons Public Domain Dedication waiver (http://creativeco mmons.org/publicdomain/zero/1.0/) applies to the data made available in this article, unless otherwise stated in a credit line to the data. 
and functional domains that cut across a number of medical and psychological conditions [2].

Initially developed and validated in the general population, PROMIS measures are increasingly being tested in clinical settings. However, there are substantial gaps in understanding the performance of PROMIS measures in patients. One particularly important psychometric characteristic is a scale's responsiveness (alternatively called sensitivity to change) which focuses on a measure's ability to detect changes over time [3]. A responsive measure is essential for clinical trials and other longitudinal studies to minimize the risk of false negative conclusions as well as to potentially reduce sample size and study costs. Responsiveness is also critical in clinical practice where the purpose is to detect clinically meaningful change over time in order to monitor and, if necessary, adjust treatment.

PROMIS measures draw upon item banks that are calibrated using item response theory and include large numbers of questions that collectively represent a welldefined, unidimensional construct. Individual questions from these large banks can then be extracted, using various strategies, to create unique short forms of that measure [2]. These short forms can be static (i.e., the same items used in a fixed-length scale), or they can be constructed adaptively in real time based on the respondent's answers to previous questions, known as computer adaptive testing (CAT). Although CAT may require a few less items than fixed-length forms to obtain comparable precision, the small increase in efficiency may not be sufficient to justify the added technical requirements for CAT administration.

Four PROMIS fixed-length depression scales are the focus of this study, which includes one with 4 items, one with 6 items, and two with 8 items. Fixed-length scales were chosen rather than CAT administration because in many clinical and research settings fixed-length scales are more feasible to administer and produce approximately comparable results to CAT. For this reason, fixed-length scales have been offered as a viable option by PROMIS developers [4].

Only a few studies have examined PROMIS depression scale responsiveness. These studies have several limitations, including studying only a single sample [5-8], no comparison to a legacy or other anchor measure[7], and focusing only or principally on CAT rather than fixedlength PROMIS measures [5, 7, 9]. Given the limitations of previous studies, our study purpose was to evaluate responsiveness of the four fixed-length PROMIS depression scales, and compare their responsiveness to legacy depression measures using three clinical samples. It should be noted that scores on these self-report scales represent depressive symptom severity rather than a depressive disorder diagnosis; the latter requires a clinical assessment.

\section{Methods \\ Design and participants}

Data were analyzed from three randomized controlled trials (RCTs) conducted between 2012 and 2017. Trial details were provided in a previous report of the minimally important differences and severity thresholds for the PROMIS depression measures [10]. Briefly, the study sample includes 651 patients who had complete psychometric data on depression measures (Table 1). Sample 1 (CAMEO trial) consisted of 153 primary care patients participating in an RCT to compare the effectiveness of pharmacological versus cognitive-behavioral treatment for chronic low back pain. Sample 2 (SPACE trial) consisted of 240 primary care patients participating in a pragmatic RCT comparing opioid therapy versus nonopioid medication therapy for chronic back pain or hip or knee osteoarthritis pain. Sample 3 (SSM trial) consisted of 258 stroke survivors participating in an RCT evaluating the efficacy of a stroke-self-management program. Samples 1 and 2 were enrolled from Veterans Administration (VA) primary care clinics, and Sample 3 comprised both Veteran and non-Veteran patients. Data were collected from baseline and follow-up interviews administered by trained research personnel. Follow-up assessments were conducted 6 months after baseline for Sample 1 and 3 months after baseline for Samples 2 and 3. The studies were approved by the Indiana University Institutional Review Board.

\section{Measures}

\section{PROMIS Depression Scales}

We evaluated four fixed-length PROMIS depression scales: the original 8-item depression Short Form (8b), and the 4-item (4a), 6-item (6a) and 8-item (8a) depression scales from the PROMIS profiles (a collection of short forms containing a fixed number of items from key PROMIS domains). Items are nested in the latter three scales: the 6a scale adds two items to the 4a scale, and the $8 \mathrm{a}$ scale adds two items to the $6 \mathrm{a}$ scale. The $8 \mathrm{a}$ and $8 \mathrm{~b}$ scales share 7 items in common and 1 unique item each. For each scale, respondents are asked how often in the past 7 days they have experienced specific depression symptoms, using a 5-point ordinal rating scale of "Never," "Rarely," "Sometimes," "Often," and "Always." Raw score totals are converted to an item response theory-based T-scores. A T-score of 50 is the average for the United States general population with a standard deviation (SD) of 10. A higher T-score represents greater depression severity. Cronbach's alphas for baseline PROMIS raw scores in the three trials ranged from 0.89 to 0.95 . 
Table 1 Characteristics of samples in three randomized controlled trials (RCTs)

\begin{tabular}{|c|c|c|c|}
\hline \multirow[t]{2}{*}{ Clinical population } & \multirow{2}{*}{$\begin{array}{l}\text { Sample } 1 \text { CAMEO RCT }\left(N_{1}=153\right) \\
\text { Chronic low back pain }\end{array}$} & \multirow{2}{*}{$\begin{array}{l}\text { Sample } 2 \text { SPACE RCT }\left(\mathrm{N}_{2}=240\right) \\
\text { Chronic musculoskeletal pain }\end{array}$} & \multirow{2}{*}{$\begin{array}{l}\text { Sample } 3 \mathrm{SSM} \\
\mathrm{RCT}\left(\mathrm{N}_{3}=258\right) \\
\text { Stroke survivor }\end{array}$} \\
\hline & & & \\
\hline Recruitment setting & Primary care & Primary care & Neurology \\
\hline Age, mean (SD) & $58.1(9.3)$ & $58.3(13.7)$ & $61.7(10.8)$ \\
\hline Male, n (\%) & $140(91.5)$ & $208(86.7)$ & $209(81.0)$ \\
\hline \multicolumn{4}{|l|}{ Race, n (\%) } \\
\hline White & $111(72.5)$ & $207(86.2)$ & $166(64.3)$ \\
\hline Black & $37(24.2)$ & $18(7.5)$ & $78(30.2)$ \\
\hline Other & $5(3.3)$ & $15(6.3)$ & $14(5.4)$ \\
\hline \multicolumn{4}{|l|}{ Education, n (\%) } \\
\hline Less than high school & $8(5.2)$ & $6(2.5)$ & $31(12.2)$ \\
\hline High school & $49(32.0)$ & $71(29.6)$ & $85(33.3)$ \\
\hline Technical school or some college & $74(48.4)$ & $103(42.9)$ & $80(31.4)$ \\
\hline College degree or greater & $22(14.4)$ & $60(25.0)$ & $59(23.1)$ \\
\hline \multicolumn{4}{|l|}{ Marital status, n (\%) } \\
\hline Married & $81(52.9)$ & $135(56.5)$ & $135(52.5)$ \\
\hline Divorced & $43(28.1)$ & $60(25.1)$ & $68(26.5)$ \\
\hline Other & $29(19.0)$ & $44(18.4)$ & $54(21.0)$ \\
\hline \multicolumn{4}{|l|}{ PROMIS T-scores, mean (SD) } \\
\hline Depression 4-item & $53.5(9.9)$ & $50.3(9.1)$ & $51.3(9.2)$ \\
\hline Depression 6-item & $53.2(10.3)$ & $49.9(9.5)$ & $50.5(10.0)$ \\
\hline Depression 8-item & $53.0(10.2)$ & $49.6(9.5)$ & $50.3(9.9)$ \\
\hline Depression short-form & $53.0(10.3)$ & $49.7(9.7)$ & $50.0(10.3)$ \\
\hline PHQ-9 depression score, mean (SD) & $11.1(6.2)$ & $6.2(5.0)$ & $7.7(6.2)$ \\
\hline $\begin{array}{l}\text { Cross-sectional Global Ratings of Depression } \\
(0-4) \text {, mean (SD) }\end{array}$ & $2.5(1.0)$ & $2.0(0.9)$ & $1.9(1.0)$ \\
\hline \multicolumn{4}{|l|}{ DSM-V depressive disorder, $\mathrm{n}(\%)$} \\
\hline Major depression & $68(44.4)$ & $36(15.0)$ & $66(25.6)$ \\
\hline Minor depression & $21(13.7)$ & $23(9.6)$ & $21(8.1)$ \\
\hline Disability days in the past 4 weeks, mean (SD) & $16.3(8.6)$ & $10.3(9.0)$ & $5.1(7.7)$ \\
\hline
\end{tabular}

CAMEO CAre Management for the Effective use of Opioids trial, SPACE Strategies for Prescribing Analgesics Comparative Effectiveness trial, SSM Stroke survivor SelfManagement trial

\section{Patient Health Questionnaire 9-item (PHQ-9) and 2-item (PHQ-2) Depression Scales}

The PHQ-9 is among the best-validated and widelyused depression scales in both clinical practice and research [11, 12]. The PHQ-9 [13] includes 1 item for each of the 9 DSM-V criterion symptoms used in diagnosing major depression. Respondents are asked how much in the past 2 weeks they have been bothered by each symptom, with the response options being "Not at all", "Several days", "More than half the days", and "Nearly every day." Scores range from 0 to 27 with higher scores indicating greater depression severity. The Cronbach's alpha for baseline PHQ-9 scores in the three trials ranged from 0.76 to 0.85 . The PHQ- 2 comprises the first two items of the PHQ-9 that capture depressed mood and anhedonia. It is scored 0 to 6 and has been validated as an ultra-brief screening tool [12] with some evidence of responsiveness $[13,14]$.

\section{SF-36 Mental Health Scale}

The SF-36 Mental Health scale was administered only in Sample 1 (CAMEO trial). The scale consists of five items with each item scored from 1 (not at all) to 5 (extremely) scale over the past four weeks. Responses from the five items are summed and then transformed to a $0-100$ scale where a lower number represents more severe symptoms. The scale has demonstrated good operating characteristics as a depression screener as well as sensitive to change in longitudinal studies $[15,16]$. 


\section{Prospective global rating of change}

The prospective global rating of change is the difference between an individual's cross-sectional global rating of mood at two time points (baseline minus follow-up) [17]. Because the cross-sectional global rating is on a 5-point scale ranging from 0 ("Not unhappy or down at all") to 4 ("Very severely unhappy or down"), change scores have a possible range of -4 to +4 , where negative numbers indicated worsening mood and positive numbers improved mood. For example, a patient who reported being "severely unhappy or down" at baseline and "mildly unhappy or down" at follow-up would have $a+2$ change $(3-1)$, whereas a patient who reported being "moderately unhappy or down" at baseline and "severely unhappy or down" at follow-up would have a -1 change $(2-3)$. Change scores were collapsed into three categories of better $(+1$ to +4$)$, same $(0)$, and worse $(-1$ to -4$)$. We used this prospective anchor to overcome potential recall and reconstruction bias related to the retrospective global rating of change [18]. A few studies have suggested, compared to the retrospective global rating of change, that the prospective global rating of change may be less influenced by post-treatment status $[18,19]$.

\section{Retrospective global rating of change}

The retrospective global rating of change assesses overall clinical response from the participant's perspective [20]. At follow-up, participants were asked to rate their mood change compared to their mood at baseline assessment. Change in mood is rated on a 7-point scale with the following response options: -3 (much worse), -2 (moderately worse), -1 (a little worse), 0 (no change),+1 (a little better), +2 (moderately better), or +3 (much better). Based on the rating, participants were further categorized into three groups, improved $(+1$ to +3$)$, unchanged ( 0$)$, and worsened $(-1$ to -3$)$. The retrospective global rating of change has been widely used to assess responsiveness of patient-reported outcome measures $[3,16]$.

\section{Statistical analysis}

We evaluated comparative responsiveness for all four PROMIS scales and legacy measures (i.e., PHQ-9, PHQ2, and SF-36 Mental Health). Data from each of the three trials were analyzed separately rather than pooled, because the three trials involved different clinical populations, study interventions, and follow-up timeframes. We used both prospective and retrospective global ratings of change for mood as the anchors (i.e., criteria) to identify patients who had changed since baseline. Specifically, patients were categorized into three groups based on global ratings of mood change: better, same, and worse.
Both within-group and between-group responsiveness to change were evaluated.

\section{Within-group responsiveness}

For within-group responsiveness, we estimated the amount of change over time within each global rating of depression change group (i.e., better, same, and worse). The standardized response mean (SRM) was used as the effect size measure of within-group responsiveness to change. The SRM is the ratio of the mean change to the standardized deviation (SD) of change, and is calculated using the formula (mean baseline score-mean followup score)/(SD of change score). We also calculated $95 \%$ confidence intervals for the SRMs with a bootstrapping procedure. SRM values of $0.2,0.5$, and 0.8 represent thresholds for small, moderate and large changes, respectively [3, 21]. Some researchers suggest an absolute SRM value $\geq 0.3$ indicates responsiveness [22].

\section{Between-group responsiveness}

For between-group responsiveness, we compared the amount of change between global rating of change groups. First, we used omnibus ANOVA tests to compare mean change scores across global rating of change groups (i.e., improved, unchanged, and worsened). For this analysis, both retrospective and prospective rating of change groups were used as anchors. We used post-hoc TukeyKramer pairwise tests to compare the three groups and controlled for family-wise Type 1 error at 0.05 .

Second, we used receiver-operating characteristic curve analyses to further quantify a measure's ability to detect improvement. Area under the curve (AUC) is the probability of correctly discriminating between patients who have improved and those who have not. The AUC values range from 0.5 (the same as chance) to 1.0 (perfect discrimination). We calculated the AUC for each depression measure using retrospective and prospective global ratings of change as the anchors. For the retrospective anchor, we evaluated each measure's ability to detect any improvement ("a little better", "moderately better", or "very much better") as well as moderate improvement ("moderately better" or "very much better"). For the prospective anchor, we evaluated each measure's ability to detect any improvement $(+1$ to +4$)$ as well as moderate improvement $(+2$ to +4$)$. To determine if depression scales differed in their ability to detect improvement, we also statistically compared AUC values between measures [20, 23].

\section{Results}

Demographic and clinical characteristics

For all three samples, participants were mostly male, non-Hispanic, white, married, and had some college 
education (Table 1). Mean PHQ-9 scores indicated that sample 1 had moderate and samples 2 and 3 had mild levels of depressive symptoms. The proportion of patients who met DSM-V criteria for major or minor depression in the 3 studies was $58.1 \%, 24.6 \%$, and $33.7 \%$, respectively.

\section{Within-group responsiveness}

In Fig. 1, within-group effect size estimates (i.e., SRMs) were plotted for the PROMIS depression and legacy measures across the three trials. This figure provides an overview of comparative within-group responsiveness across the depression measures. Tables 2 and 3 complement Fig. 1 by presenting the unstandardized change scores and SRMs with confidence intervals for the prospective and retrospective anchors, respectively.

Across the PROMIS depression, PHQ-9 and PHQ-2 scales, the SRM point estimates were generally similar (Figs. 1). In most cases, the confidence interval for one measure included the point estimates of the other measures (Tables 1 and 2), which indirectly suggests statistically comparable within-group responsiveness across these three measures. SRMs for the SF-36 mental health scale differed somewhat from the other measures, although data for this scale was only available from one trial.
Minor differences in SRMs, however, were observed. For example, retrospective anchor analyses in the CAMEO trial (sample 1) found larger absolute SRMs for improvement with the PHQ-9 compared to PROMIS but larger SRMs for worsening with the PROMIS. In contrast, the SSM trial (sample 3) revealed larger SRMs for worsening with the PHQ-9 and PHQ-2.

Across the four PROMIS depression scales of varying lengths, the SRMs were relatively comparable (Fig. 2). The mean (median) within-group difference in SRMs between any two PROMIS scales was $0.084(0.080)$ using the prospective anchor and within $0.114(0.070)$ using the retrospective anchor. Because the SRM estimates for the four PROMIS scales were similar, we reported averages of SRMs across the four PROMIS depression short forms in Fig. 1.

\section{Between-group responsiveness}

As shown in Table 2, all measures successfully detected differences among depression improved, unchanged, and worsened groups when classified by the prospective global rating of change for mood. Omnibus F-tests were all significant (many at $p<0.0001$ ) for overall differentiation among change with only one exception (the PHQ-2 in the CAMEO trial). In pair-wise comparisons,

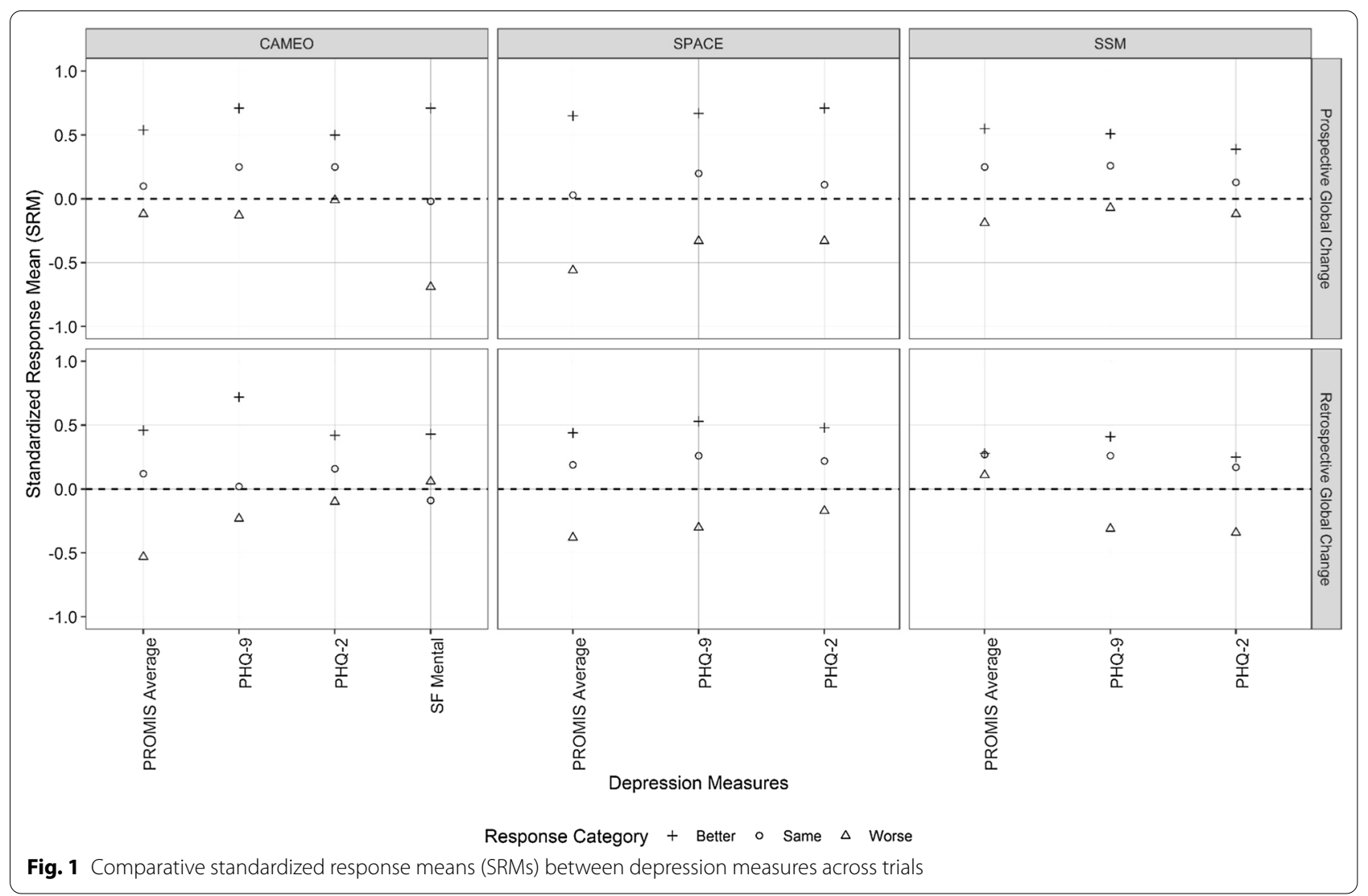




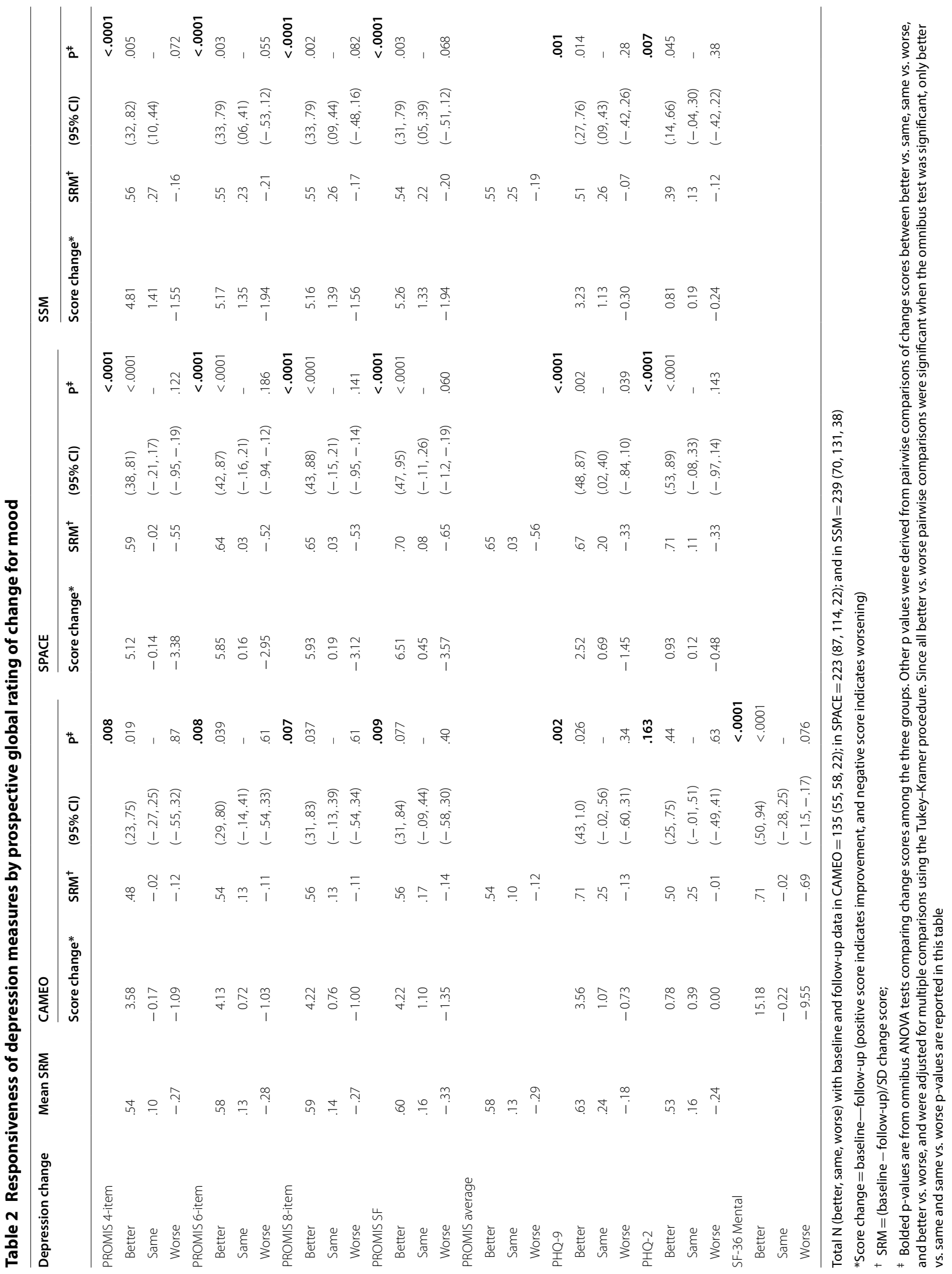




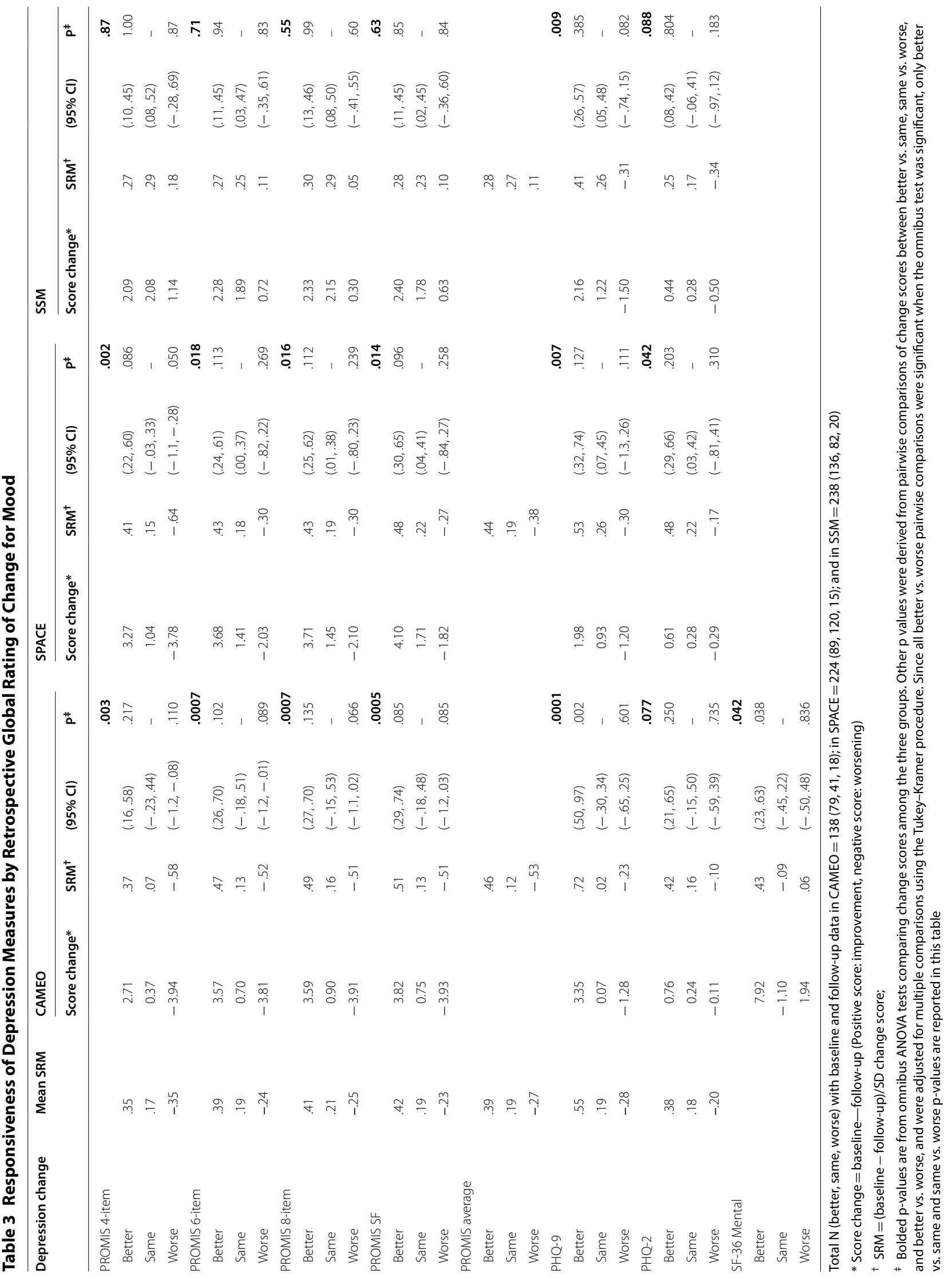




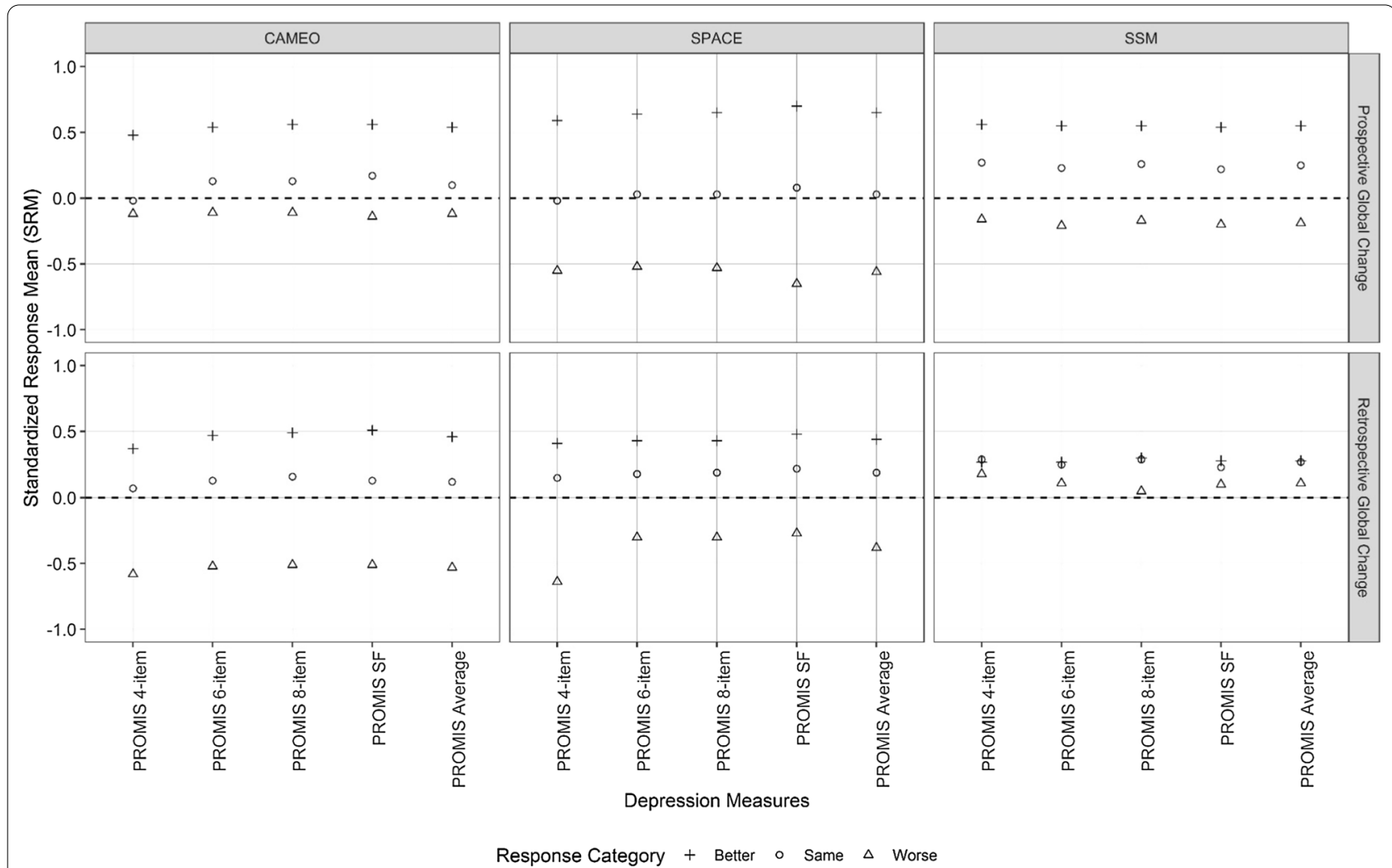

Fig. 2 Comparative standardized response means (SRMs) between PROMIS depression short forms of varying lengths

scales distinguished better from unchanged in all but two instances (the PROMIS short-form $8 \mathrm{~b}$ and PHQ-2 in the CAMEO trial). In contrast, scales did not distinguish worse from unchanged except in one instance (the PHQ-9 in the SPACE trial). The mean SRM for the PROMIS average, PHQ-9, and PHQ-2 scores across the CAMEO, SPACE and SSM trials was 0.58, 0.63, and 0.53 for the improved group; $0.13,0.27$, and 0.16 for the unchanged group; and $-0.29,-0.18$, and -0.15 for the worse group.

When using the retrospective global rating of change anchor (Table 3), the ability of measures to detect differences among the three groups was not quite as strong. Omnibus F-tests were still significant in two of the trials (except the PHQ-2 in CAMEO) but not as highly significant as for the prospective anchor. Moreover, none of the omnibus F-tests were significant in the SSM trial, except for the PHQ-9. The mean SRM using the retrospective anchor for the PROMIS average, PHQ-9, and PHQ-2 scores across the CAMEO, SPACE and SSM trials was $0.39,0.55$, and 0.38 for the improved group; $0.19,0.18$, and 0.18 for the unchanged group; and $-0.27,-0.28$, and -0.20 for the worse group.

Table 4 shows the results from the AUC analysis for moderate improvement. Averaged across the 3 trials,
AUCs using the retrospective global change anchor were 0.603 to 0.625 for the PROMIS scales, 0.636 for the PHQ-9, and 0.588 for the PHQ-2. AUCs using the prospective global change anchor averaged $0.745-0.757$ for the PROMIS scales, 0.682 for the PHQ-9, and 0.631 for the PHQ-2. Table 5 shows that AUCs for detecting any improvement were somewhat lower.

\section{Agreement between retrospective and global rating of change anchors}

The retrospective and prospective global change anchors agreed in their categorization of individuals as better, same, or worse in 68 of 136 participants in CAMEO, 123 of 223 in SPACE, and 95 of 238 in SSSM, resulting in simple agreement rates of $50 \%, 55 \%$, and $40 \%$ respectively. The corresponding weighted kappas in the 3 trials were $0.228,0.233$, and -0.027 .

\section{Discussion}

Using data from three clinical trials, we found PROMIS depression scales were responsive to change using both prospective and retrospective global change anchors as well as AUC analysis. Responsiveness was similar among all four fixed-length PROMIS scales and comparable to the responsiveness of the PHQ-9 and PHQ-2. In 


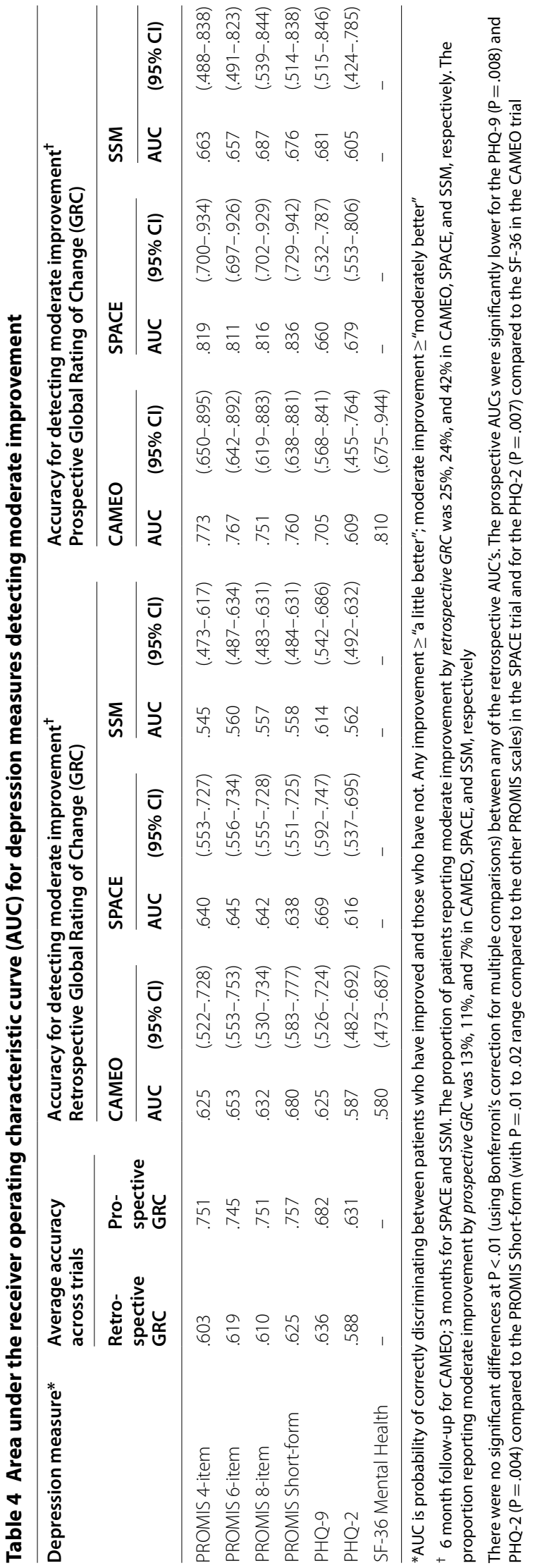




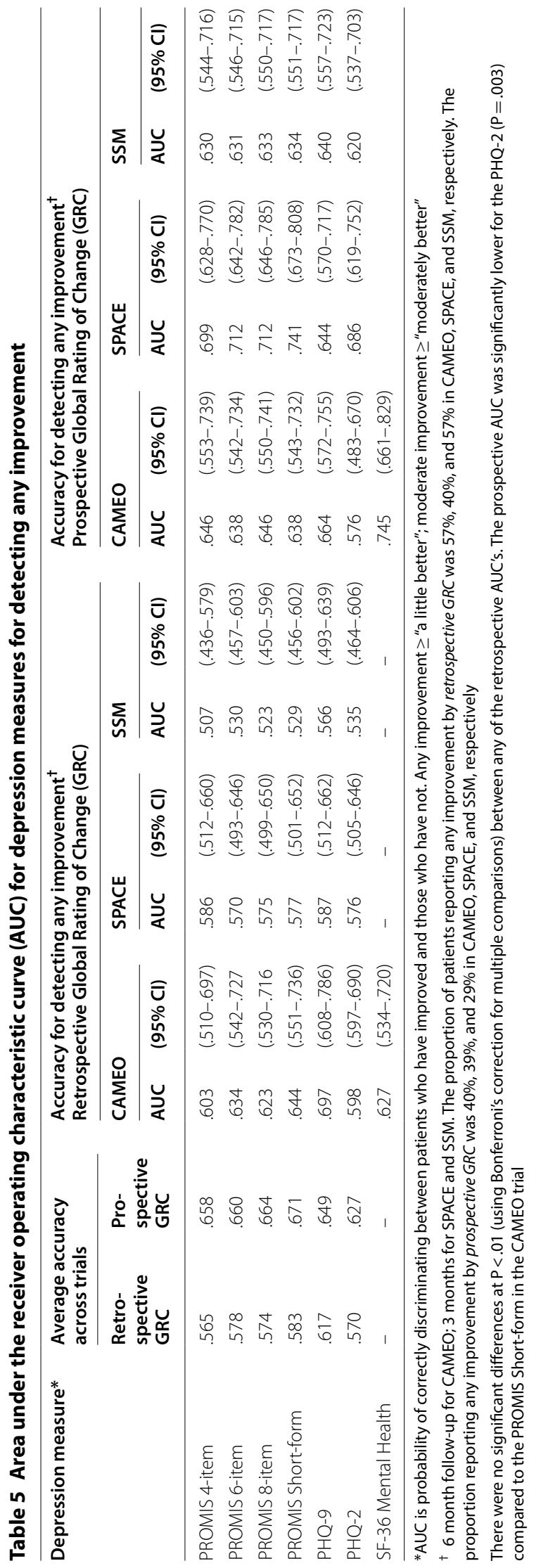


general, the measures were better able to detect depression improvement than worsening. A strength of our study compared to previous research on responsiveness of PROMIS depression measures is the triangulation of results from three patient samples using three measures of responsiveness.

Only a few prior studies have explored the responsiveness of PROMIS depression scales. In an observational study of 234 patients undergoing inpatient treatment in four psychosomatic rehabilitation centers, the prepost treatment effect size was similar for the PROMIS depression item bank scale (using all 28 items) and the Center for Epidemiological Studies Depression scale (CES-D) (1.16 vs. 1.09) [7]. In a second observational study of 194 patients with depression treated for 12 weeks, the PROMIS CAT was similar to the PHQ-9 and CES-D in terms of treatment effect size: 0.84, 0.98, and 1.06, respectively [5]. However, depression recovery defined in several different ways was less frequent with the PHQ-9 compared to PROMIS and CES-D. In contrast, the PHQ-9 and PROMIS 8-item short-form had similar responsiveness in identifying depression recovery in a longitudinal study of 701 patients with neurological or psychiatric disorders [8]. In a longitudinal study of 903 patients with 5 diverse diseases (4 medical conditions and major depressive disorder), two thirds of patients completed PROMIS by CAT and one-third with an 8-item short form [9]. The average SRM using a retrospective global anchor was 0.71 for the improved group and -0.49 for the group that worsened. In a longitudinal study of 150 patients with depression, SRMs in those experiencing recovery were 0.82 and 0.79 for the PROMIS 28-item bank and 8-item short form depression scales, respectively, and 1.00 for the PHQ-9 [6]. Unlike these previous studies that used either an observational design, a single sample, or PROMIS administration by CAT or the entire item bank, we used data from three RCTs and evaluated four PROMIS short forms of varying lengths. In addition, we evaluated responsiveness by triangulating several methods. Thus, our study substantially strengthens the evidence regarding the responsiveness of PROMIS depression scales.

Responsiveness was not symmetric with respect to improvement and worsening. SRMs for improvement averaged a moderate positive effect size and were roughly twice the SRMs for worsening which averaged a small negative effective size. Also, the 3 to 6 point improvement in PROMIS depression T-scores was above the minimally important difference. This greater sensitivity of symptom scales for detecting improvement has been previously reported for depression $[5,16,24]$, pain $[20$, $22,25-28]$ and anxiety [24].
The Consensus-based Standards for the selection of health Measurement Instruments (COSMIN) guidelines consider SRMs and other effect size metrics an imperfect approach to assessing responsiveness [29] and also discuss the limitations of transition anchors such as global rating of change. Objections to these opinions [30] as well as the COSMIN rationale [31] have been subsequently articulated. Suffice it to say, SRMs and effect sizes as well as global of rating change anchors have been widely used to assess responsiveness both before [3, 30, 32-34] and since [20,35-42] publication of the COSMIN guidelines; only a small number of representative studies are cited here.

The AUCs in Table 4 represent modest rather than strong differentiation between patients whose depression had improved and those who were the same or worse. However, AUCs have been reported in a similar range in other studies using retrospective global rating of change as an anchor [16, 20, 27, 43] in which AUCs tend to be lower than in studies of diagnostic tests for which there is a criterion ("gold") standard to determine the presence of a disease. Retrospective global ratings of change may be influenced by recall bias as well as the current state of symptoms [19,44]. Some experts recommend an AUC $\geq 0.70$ as a threshold for responsiveness when using a criterion standard anchor but also acknowledge that criterion standards often do not exist for patient-reported outcomes (PROs) $[29,45]$. Thus, AUCs for scales measuring symptoms and other PROs have been $<0.70$ not only when using retrospective global change anchors but also in some studies using other anchors as well [32, 46, 47]. Ours is the first study to also use prospective global change anchors to assess AUCs for PRO scales. Although this anchor lead to more AUC estimates $\geq 0.70$, the sample size of those with moderate change by this anchor was small yielding wide confidence intervals. For all these reasons, the similarity of AUCs when using a global change anchors is more salient than their absolute value [48].

Scale length did not have a strong effect on responsiveness. The four PROMIS depression scales ranging from 4 to 8 items had similar responsiveness, a finding previously reported for PROMIS pain scales [20]. The PROMIS fixed-length scales for a specific domain share some items in common, which may explain in part their comparable responsiveness. Also, the average responsiveness of the PHQ-9 and PHQ-2 did not differ substantially, as has been shown in only one previous study [13]. Short measures may be more desirable for studies with many outcome measures, particularly where depression is a secondary rather than primary outcome, or in busy clinical practice settings with time constraints or the need to assess multiple patient-reported outcome measures. 
Methodologically, our study is relatively unique in using both retrospective and prospective global change anchors allowing assessment of responsiveness with two different global anchors. Notably, two of the trials only showed fair agreement beyond chance of these two anchors in classifying individuals as better, same or worse, and one trial showed poor to no agreement beyond chance. It is possible that the two anchors provide different perspectives of change over time. Alternatively, it may be that one anchor is superior to another or that both anchors have limitations, but this would require additional research comparing both anchors to a third independent anchor. However, as already discussed, criterion standard anchors for patient-reported outcomes are lacking. Moreover, global rating of change is among one of the most commonly-used anchors for assessing responsiveness.

Our study has several limitations. First, depression was generally mild in all three samples, thereby restricting the range in which depression improvement could be detected. Responsiveness needs to be further studied in more clinically depressed samples in which treatment is warranted and a responsive measure is especially important. Second, because the samples included predominantly male veterans with either chronic pain or stroke, findings need to be replicated in populations with more women and a broader range of medical and mental health conditions. Third, one legacy measure (SF-36 Mental Health) was used only in one trial (CAMEO). Although its responsiveness has been demonstrated in prior studies, its comparative responsiveness to the PHQ-9 and PROMIS scales requires additional testing. Fourth, because we made multiple statistical comparisons between depression measures, the differences between measures should be interpreted cautiously unless highly significant (i.e., $\mathrm{p}<0.001$ ). Fifth, the nested nature of the PROMIS scales (i.e., sharing many items in common) as well as the PHQ-2 items being included in the PHQ-9 would lead to some convergence of responsiveness within the same family of scales. Sixth, studies using additional responsiveness metrics besides SRMs anchored to global ratings of change are warranted. Finally, our findings are derived from secondary analyses of data from clinical trials rather than a primary hypothesis-driven psychometric study.

\section{Conclusions}

Two well-validated and widely-used depression measures-the PHQ-9 and PROMIS scales-have generally comparable responsiveness. Moreover, the shorter versions of these scales also appear responsive. Our findings provide initial evidence of responsiveness which should be further tested in other patient samples using additional responsiveness metrics. The fact that both measures are public domain and available in numerous translations are additional advantages. Because measures seem better in detecting improvement than worsening, calculating the change in score together with a single question about global change may be desirable to optimize recognition of deterioration in symptom-based conditions like depression and pain. Recent initiatives to incorporate depression and other patient-reported outcome measures into routine practice as well as embedding them in the electronic health record will further enhance symptom detection and management.[49].

\section{Acknowledgements}

Not applicable.

\section{Authors' contributions}

KK, JK and POM designed the study, TMD, MBJ and EEK collected data, KK, TES, JK and POM nalyzed the data, KK and CXC drafted the manuscript. All authors contributed to interpretation of data, critically reviewed the manuscript, and approved the final version. All authors read and approved the final manuscript.

\section{Funding}

This work was supported by a National Institute of Arthritis and Musculoskeletal Disorders R01 award to (R01 AR064081) and Department of Veterans Affairs Health Services Research and Development Merit Review awards (IIR 10-128 and IIR 11-125), and VA HSRD QUERI Service Directed Project SDP-10379). Additional support was provided by the National Institute of Nursing Research under award number 5T32 NR007066, the Indiana University-Purdue University Indianapolis Enhanced Mentoring Program with Opportunities for Ways to Excel in Research (EMPOWER) Grant, KL2TR001106 and UL1TR001108 grants funded by the National Center for Advancing Translational Sciences Clinical and Translational Sciences Award, and Department of Veterans Affairs Rehabilitation Research and Development Career Development Award (IK2RX000879). The content is solely the responsibility of the authors and does not necessarily represent the official views of the National Institutes of Health or the Department of Veteran Affairs.

Availability of data and materials

Datasets and analyses codes generated during the current study are available from the corresponding author on reasonable request.

\section{Ethics approval and consent to participate}

The study was approved by the Indiana University, Indianapolis Veteran Affairs (VA), and Minneapolis VA institutional review boards. All participants provided written informed consent.

\section{Consent for publication}

Not applicable.

\section{Competing interests}

The authors report no competing interests.

\section{Author details \\ 1 Department of Medicine, Indiana University School of Medicine, Indianapolis, IN, USA. ${ }^{2}$ Regenstrief Institute, Inc, 1101 West 10th St., Indianapolis, IN 46202, USA. ${ }^{3}$ Department of Biostatistics, Indiana University Fairbanks School of Pub- lic Health and School of Medicine, Indianapolis, IN, USA. ${ }^{4}$ Indiana University School of Nursing, Indianapolis, IN, USA. ${ }^{5}$ Department of Population Health Sciences, University of Utah School of Medicine, Salt Lake City, Utah, USA. ${ }^{6}$ VA Health Services Research and Development Center for Health Information and Communication, Indianapolis, IN, USA. ${ }^{7}$ Center for Chronic Disease Out- comes Research, Minneapolis VA Health Care System, Minneapolis, MN, USA. ${ }^{8}$ University of Minnesota Medical School, Minneapolis, MN, USA.}


Received: 7 June 2020 Accepted: 11 January 2021

Published online: 04 February 2021

\section{References}

1. O'Connor E, Rossum RC, Henninger M, Groom HC, Burda BU, Henderson JT, Bigler KD, Whitlock EP: Screening for Depression in Adults: An Updated Systematic Evidence Review for the U.S. Preventive Services Task Force. Evidence Synthesis No. 128. Rockville, MD; 2016.

2. Cella D, Riley W, Stone A, Rothrock N, Reeve B, Yount S, Amtmann D, Bode R, Buysse D, Choi S, et al. The Patient-Reported Outcomes Measurement Information System (PROMIS) developed and tested its first wave of adult self-reported health outcome item banks: 2005-2008. J Clin Epidemiol. 2010;63:1179-94

3. Revicki D, Hays RD, Cella D, Sloan J. Recommended methods for determining responsiveness and minimally important differences for patientreported outcomes. J Clin Epidemiol. 2008:61:102-9.

4. Choi SW, Reise SP, Pilkonis PA, Hays RD, Cella D. Efficiency of static and computer adaptive short forms compared to full-length measures of depressive symptoms. Qual Life Res. 2010;19:125-36.

5. Pilkonis PA, Yu L, Dodds NE, Johnston KL, Maihoefer CC, Lawrence SM. Validation of the depression item bank from the Patient-Reported Outcomes Measurement Information System (PROMIS) in a three-month observational study. J Psychiatr Res. 2014;56:112-9.

6. Vilagut G, Forero CG, Adroher ND, Olariu E, Cella D, Alonso J. investigators IN: Testing the PROMIS(R) Depression measures for monitoring depression in a clinical sample outside the US. J Psychiatr Res. 2015;68:140-50.

7. Jakob T, Nagl M, Gramm L, Heyduck K, Farin E, Glattacker M. Psychometric Properties of a German Translation of the PROMIS(R) Depression Item Bank. Eval Health Prof. 2017:40:106-20.

8. Katzan IL, Fan Y, Griffith SD, Crane PK, Thompson NR, Cella D. Scale linking to enable patient-reported outcome performance measures assessed with different patient-reported outcome measures. Value Health. 2017:20:1143-9.

9. Schalet BD, Pilkonis PA, Yu L, Dodds N, Johnston KL, Yount S, Riley W, Cella D. Clinical validity of PROMIS Depression, Anxiety, and Anger across diverse clinical samples. J Clin Epidemiol. 2016;73:119-27.

10. Kroenke K, Stump TE, Chen CX, Kean J, Bair MJ, Damush TM, Krebs EE, Monahan PO. Minimally important differences and severity thresholds are estimated for the PROMIS depression scales from three randomized clinical trials. J Affect Disord. 2020;266:100-8.

11. Kroenke K, Spitzer RL, Williams JB, Lowe B. The Patient Health Questionnaire Somatic, Anxiety, and Depressive Symptom Scales: a systematic review. Gen Hosp Psychiatry. 2010;32:345-59.

12. Mitchell AJ, Yadegarfar M, Gill J, Stubbs B. Case finding and screening clinical utility of the Patient Health Questionnaire (PHQ-9 and PHQ-2) for depression in primary care: a diagnostic meta-analysis of 40 studies. BJPsych Open. 2016;2:127-38.

13. Lowe B, Kroenke K, Grafe K. Detecting and monitoring depression with a two-item questionnaire (PHQ-2). J Psychosom Res. 2005;58:163-71.

14. Staples LG, Dear BF, Gandy M, Fogliati V, Fogliati R, Karin E, Nielssen O, Titov N. Psychometric properties and clinical utility of brief measures of depression, anxiety, and general distress: the PHQ-2, GAD-2, and K-6. Gen Hosp Psychiatry. 2019;56:13-8.

15. Berwick DM, Murphy JM, Goldman PA, Ware JE Jr, Barsky AJ, Weinstein MC. Performance of a five-item mental health screening test. Med Care. 1991;29:169-76.

16. Johns SA, Kroenke K, Krebs EE, Theobald DE, Wu J, Tu W. Longitudinal comparison of three depression measures in adult cancer patients. J Pain Symptom Manag. 2013;45:71-82.

17. Yost KJ, Eton DT, Garcia SF, Cella D. Minimally important differences were estimated for six Patient-Reported Outcomes Measurement Information System-Cancer scales in advanced-stage cancer patients. J Clin Epidemiol. 2011;64:507-16.

18. Schmitt J, Di Fabio RP. The validity of prospective and retrospective global change criterion measures. Arch Phys Med Rehabil. 2005;86:2270-6.

19. Fletcher KE, French CT, Irwin RS, Corapi KM, Norman GR. A prospective global measure, the Punum Ladder, provides more valid assessments of quality of life than a retrospective transition measure. J Clin Epidemiol. 2010:63:1123-31.
20. Chen CX, Kroenke K, Stump T, Kean J, Krebs EE, Bair MJ, Damush T, Monahan PO. Comparative responsiveness of the PROMIS pain interference short forms with legacy pain measures: results from three randomized clinical trials. J Pain. 2019;20:664-75.

21. Kazis LE, Anderson JJ, Meenan RF. Effect sizes for interpreting changes in health status. Med Care. 1989;27:S178-89.

22. Askew RL, Cook KF, Revicki DA, Cella D, Amtmann D. Evidence from diverse clinical populations supported clinical validity of PROMIS pain interference and pain behavior. J Clin Epidemiol. 2016;73:103-11.

23. DeLong ER, DeLong DM, Clarke-Pearson DL. Comparing the areas under two or more correlated receiver operating characteristic curves: a nonparametric approach. Biometrics. 1988;44:837-45.

24. Kroenke K, Baye F, Lourens SG. Comparative validity and responsiveness of PHQ-ADS and other composite anxiety-depression measures. J Affect Disord. 2019;246:437-43.

25. Krebs EE, Bair MJ, Wu J, Damush TM, Tu W, Kroenke K. Comparative responsiveness of pain outcome measures among primary care patients with musculoskeletal pain. Med Care. 2010;48:1007-14.

26. Kroenke K, Theobald D, Wu J, Tu W, Krebs EE. Comparative responsiveness of pain measures in cancer patients. J Pain. 2012;13:764-72.

27. Kean J, Monahan PO, Kroenke K, Wu J, Yu Z, Stump TE, Krebs EE. Comparative Responsiveness of the PROMIS Pain Interference Short Forms, Brief Pain Inventory, PEG, and SF-36 Bodily Pain Subscale. Med Care. 2016;54:414-21.

28. Deyo RA, Katrina R, Buckley DI, Michaels L, Kobus A, Eckstrom E, Forro V, Morris C. Performance of a Patient Reported Outcomes Measurement Information System (PROMIS) Short Form in older adults with chronic musculoskeletal pain. Pain Med. 2015;17:314-24.

29. Mokkink LB, Terwee CB, Knol DL, Stratford PW, Alonso J, Patrick DL, Bouter $L M$, de Vet HC. The COSMIN checklist for evaluating the methodological quality of studies on measurement properties: a clarification of its content. BMC Med Res Methodol. 2010;10:22.

30. Angst F: The new COSMIN guidelines confront traditional concepts of responsiveness. BMC Med Res Methodol 2011, 11:152; author reply 152.

31. Mokkink LB, Terwee CB, Knol DL, de Vet HC. The new COSMIN quidelines regarding responsiveness. Author's response. BMC Med Res Methodol. 2011:11:152.

32. Husted JA, Cook RJ, Farewell VT, Gladman DD. Methods for assessing responsiveness: a critical review and recommendations. J Clin Epidemiol. 2000;53:459-68.

33. Middel B, van Sonderen E. Statistical significant change versus relevant or important change in (quasi) experimental design: some conceptual and methodological problems in estimating magnitude of interventionrelated change in health services research. Int J Integr Care. 2002;2:e15.

34. Norman GR, Wyrwich KW, Patrick DL. The mathematical relationship among different forms of responsiveness coefficients. Qual Life Res. 2007;16:815-22.

35. Jensen RE, Moinpour CM, Potosky AL, Lobo T, Hahn EA, Hays RD, Cella D, Smith AW, Wu XC, Keegan TH, et al. Responsiveness of 8 Patient-Reported Outcomes Measurement Information System (PROMIS) measures in a large, community-based cancer study cohort. Cancer. 2017;123:327-35.

36. Cella D, Lai JS, Jensen SE, Christodoulou C, Junghaenel DU, Reeve BB, Stone AA. PROMIS fatique item bank had clinical validity across diverse chronic conditions. J Clin Epidemiol. 2016;73:128-34.

37. Cook KF, Jensen SE, Schalet BD, Beaumont JL, Amtmann D, Czajkowski $\mathrm{S}$, Dewalt DA, Fries JF, Pilkonis PA, Reeve BB, et al. PROMIS measures of pain, fatigue, negative affect, physical function, and social function demonstrated clinical validity across a range of chronic conditions. J Clin Epidemiol. 2016;73:89-102.

38. Schalet BD, Hays RD, Jensen SE, Beaumont JL, Fries JF, Cella D. Validity of PROMIS physical function measured in diverse clinical samples. J Clin Epidemiol. 2016;73:112-8.

39. Hung M, Saltzman CL, Greene T, Voss MW, Bounsanga J, Gu Y, Anderson $\mathrm{MB}$, Peters $\mathrm{CL}$, Gililland J, Pelt CE. Evaluating instrument responsiveness in joint function: The HOOS JR, the KOOS JR, and the PROMIS PF CAT. J Orthop Res. 2018:36:1178-84.

40. Hinds PS, Wang J, Cheng YI, Stern E, Waldron M, Gross H, DeWalt DA, Jacobs SS. PROMIS pediatric measures validated in a longitudinal study design in pediatric oncology. Pediatr Blood Cancer. 2019;66:e27606. 
41. Kroenke K, Baye F, Lourens SG. Comparative responsiveness and minimally important difference of common anxiety measures. Med Care. 2019;57:890-7.

42. Katz P, Pedro S, Alemao E, Yazdany J, Dall'Era M, Trupin L, Rush S, Michaud K. Estimates of responsiveness, minimally important differences, and patient acceptable symptom state in five patient-reported outcomes measurement information system short forms in systemic lupus erythematosus. ACR Open Rheumatol. 2020;2:53-60.

43. Bushey MA, Kroenke K, Baye F, Lourens S. Assessing depression improvement with the remission evaluation and mood inventory tool (REMIT) Gen Hosp Psychiatry. 2019;60:44-9.

44. Norman GR, Stratford P, Regehr G. Methodological problems in the retrospective computation of responsiveness to change: the lesson of Cronbach. J Clin Epidemiol. 1997;50:869-79.

45. Prinsen CAC, Mokkink LB, Bouter LM, Alonso J, Patrick DL, de Vet HCW, Terwee CB. COSMIN guideline for systematic reviews of patient-reported outcome measures. Qual Life Res. 2018;27:1147-57.

46. Deyo RA, Centor RM. Assessing the responsiveness of functional scales to clinical change: an analogy to diagnostic test performance. J Chronic Dis. 1986:39:897-906.
47. Turner D, Schunemann HJ, Griffith LE, Beaton DE, Griffiths AM, Critch JN, Guyatt $\mathrm{GH}$. Using the entire cohort in the receiver operating characteristic analysis maximizes precision of the minimal important difference. $J$ Clin Epidemiol. 2009;62:374-9.

48. Deyo RA, Diehr P, Patrick DL. Reproducibility and responsiveness of health status measures. Statistics and strategies for evaluation. Control Clin Trials. 1991;12:142S-158S

49. Wu AW, Kharrazi H, Boulware L, Snyder CF. Measure once, cut twice-adding patient-reported outcome measures to the electronic health record for comparative effectiveness research. J Clin Epidemiol. 2013;8:S12-20.

\section{Publisher's Note}

Springer Nature remains neutral with regard to jurisdictional claims in published maps and institutional affiliations.
Ready to submit your research? Choose BMC and benefit from:

- fast, convenient online submission

- thorough peer review by experienced researchers in your field

- rapid publication on acceptance

- support for research data, including large and complex data types

- gold Open Access which fosters wider collaboration and increased citations

- maximum visibility for your research: over $100 \mathrm{M}$ website views per year

At BMC, research is always in progress.

Learn more biomedcentral.com/submissions 